\title{
LOS MOVIMIENTOS \\ POR LA SOLIDARIDAD; ¿UN NUEVO MODELO DE ACCIÓN COLECTIVA?
}

\author{
Pedro Ibarra
}

Universidad del País Vasco

\begin{abstract}
RESUMEN
Este texto sostiene que aquellos movimientos sociales que tienen la solidaridad como objetivo - cooperación con el Tercer Mundo, antirracismo, etc.- muestran ciertas pautas de conducta diferentes respecto a las que caracterizan al conjunto de los movimientos sociales. Asimismo, se formula en él la hipótesis que estas distintas formas de acción son también utilizadas en otras demandas sociales (ámbito del trabajo, la ecología, etc.) y que, por lo tanto, el objeto de estudio no es sólo un específico movimiento social, sino quizás una nueva y genérica forma de acción colectiva.
\end{abstract}

\section{INTRODUCCIÓN}

\section{A) Hacia un NUEVo MOdelo de ACCión COLECTIVA}

Afirmar que nos hallamos ante la aparición de un nuevo modelo de acción colectiva supone un notable "atrevimiento", por lo que resulta previo a cualquier otra consideración insistir que nos movemos en un terreno estrictamente hipotético. El autor dirige desde hace un cierto tiempo una investigación sobre determinados movimientos sociales. En esta investigación aparecen hechos, actitudes, creencias, etc., de los que pudiera deducirse que nos hallamos ante el surgimiento de tal nuevo modelo, al que luego haremos referencia. La deduc- 
ción, por tanto, es condicional. La muestra analizada es todavía insuficiente; la investigación tiene que extenderse a otros movimientos sociales, a otros espacios de acción colectiva, debe ser comparada con movimientos de otros territorios y, finalmente, hay que ampliar sus herramientas analíticas ${ }^{1}$. Ello supone que todavía no estamos en situación de poder probar empíricamente nuestra propuesta. Por el momento, nos movemos en un nivel fundamentalmente intuitivo, pendiente de ser confirmado —o también, por supuesto, negado- por la realidad.

Este trabajo se enmarca originalmente en el desarrollo de una investigación empírica en torno al grupo específico de movimientos que denominaremos como movimientos por la solidaridad (cooperación con el Tercer Mundo, antirracismo, etc.), que serán detalladamente descritos más adelante. A medida que nuestra labor progresaba, detectamos la existencia de diferencias relevantes con respecto a los demás movimientos sociales. Estos rasgos diferenciadores radican no sólo en lo distinto de sus objetivos, sino también en cómo son perseguidos los mismos. Sin embargo, y aquí introducimos una nueva y aún más arriesgada hipótesis, tras ampliar la muestra empírica, descubrimos que algunas otras acciones colectivas van adquiriendo formas similares a las de los movimientos por la solidaridad. En otros términos, descubrimos que, por ejemplo, movimientos ecologistas o pacifistas, $\mathrm{u}$ otros vinculados a la esfera del mundo del trabajo, comenzaban su andadura eligiendo las formas de ser y de actuar de los movimientos por la solidaridad. Este nuevo escenario es lo que nos mueve a considerar la posibilidad de que lo que estamos observando es un nuevo modelo de acción colectiva; nuevas formas de responder a conflictos y tensiones que en el pasado eran canalizados a través de movimientos sociales o grupos de presión. En cualquier caso, concentraremos nuestra propuesta analítica en las características especiales de los movimientos por la solidaridad y sólo al final nos arriesgaremos a sugerir propuestas en la línea de esa supuestamente nueva - y extendida - forma de acción colectiva.

\section{B) LOS MOVIMIENTOS POR LA SOLIDARIDAD}

En la categoría que denominamos como movimientos por la solidaridad se incluyen movimientos de apoyo a los emigrantes, movimientos antirracistas, de solidaridad con el Tercer Mundo (o movimientos de cooperación al desarrollo) y, en menor medida, movimientos de apoyo a grupos sociales marginados (lucha contra la pobreza) o a grupos de minusválidos; algunas agrupaciones pacifistas habrían de ser asimismo incluidas en este conjunto; en concreto, aquellas que se movilizan por las acciones terroristas de ETA.

${ }^{1}$ La investigación de momento se halla limitada al País Vasco, aunque también hemos accedido a estudios y realizado algunas entrevistas correspondientes a otras Comunidades Autónomas. Su extensión exige, por tanto, ampliar los datos en esas Comunidades y también establecer procesos comparativos con otros países europeos. Asimismo, debemos reforzar nuestros métodos cualitativos de entrevistas con algunos acercamientos más cuantitativos. 
La característica más visible de los grupos citados consiste en que para ellos la solidaridad es no sólo la forma o el medio de actuar colectivamente, sino que supone también, y sobre todo, la meta de su movilización. En efecto, tales movimientos actúan en beneficio de otros grupos, colectivos o categorías de individuos; esta característica denota también, aunque de una forma menos obvia, el carácter de los grupos pacifistas antiterroristas, para los que el objetivo -en última instancia - es una demanda de respeto por las vidas de otros, por las potenciales víctimas del terrorismo. Atendiendo a este propósito, parece adecuado denominarlos también como movimientos por la solidaridad. En cualquier caso, y como veremos, los rasgos más originales de estos movimientos, el conjunto de características que motivan las hipótesis que serán desarrolladas a través de este artículo, no están tanto relacionados con el contenido de sus demandas como con ciertos aspectos de su identidad, estrategia y organización.

Ciertamente y desde una perspectiva puramente temporal, este conjunto de movimientos no es algo nuevo ni reciente. Tanto en Europa como en España han estado desarrollando su actividad pública desde hace muchos años. La novedad, al menos en el caso español, radica en la espectacular expansión que han alcanzado en los años recientes. Este conjunto de movimientos por la solidaridad ha logrado tal grado de desarrollo y un nivel de reconocimiento social tal, que la opinión pública tiende a confundirles con la globalidad de los movimientos sociales. Es decir, en el frame del discurso social (Laclau, 1990) o a través de los master frames existentes en la realidad (Snow y Benford, 1992), estos movimientos por la solidaridad son los movimientos sociales reales. Creadores y líderes de la opinión pública les otorgan el papel de expresar la "auténtica» sociedad, esa parte de la última que no ha capitulado a la cultura de irresponsabilidad, atomización y soledad generada por los excesos y efectos perversos del Estado de Bienestar².

\section{C) MARCO ANALÍTICO}

Es nuestra intención categorizar estos movimientos a través de un marco analítico no demasiado habitual: por medio de los diferentes procesos de institucionalización a los que están sujetos los diferentes movimientos sociales.

Es evidente que lo que define a los movimientos sociales en su origen, constitución y consolidación es precisamente su carácter antiinstitucional (o al menos no institucional). Desde los movimientos sociales se afirma que buscan el conflicto con las instituciones políticas y que su organización, la de los movimientos, no se corresponde por tanto con la de otras instituciones que

2 Éstos son los movimientos más citados y más elogiados en los medios de comunicación; asimismo, cuando los líderes políticos cantan las excelencias de la sociedad civil, ponen habitualmente el ejemplo de esos miles de personas que dedican su tiempo a ayudar a los demás. 
existen de facto en la sociedad (partidos políticos, las diversas iglesias o las corporaciones económicas); ni siquiera — se afirma— se «sienten» como una institución.

Tales afirmaciones, frecuentemente implícitas, son ciertas. Sin embargo, es verdad también que, desde un cierto punto de vista, un movimiento social es una institución. Aunque desarrollaremos más abajo esta puntualización, afirmamos ahora que una institución —cualquiera de ellas, empezando por la familia, pasando por la forma del lenguaje y terminando por el partido político- es un conjunto de normas preestablecidas, que se derivan de la sedimentación de una práctica histórica, y que formal e informalmente constituye una guía para la acción.

Desde esta perspectiva, un movimiento social es también una institución y, como veremos, adopta esas normas preestablecidas que llamamos formalinstitución para fortalecer su capacidad de lograr —en contra de las instituciones políticas - ciertas transformaciones sociales.

Esta definición de forma/institución no difiere de la que pueda corresponder al concepto de cultura, entendiendo ésta de una forma dinámica e instrumental (Swindler, 1986), pero parece más adecuado utilizar el término institución ya que nos permite comparar los movimientos por la solidaridad con el resto de movimientos sociales. Efectivamente, podemos observar que tanto unos como otros adoptan la misma forma/institución, pero no son idénticos (e incluso en algunos casos decididamente diferentes) en las funciones institucionales que desarrollan.

Dicho de otra forma. Todas las opciones estables de acción colectiva son instituciones y a todas ellas es de aplicación el concepto de forma/institución que ahora veremos. Pero no todas esas opciones tienen las mismas funciones institucionales. Ello quiere decir que los grupos que vamos a analizar, definidos en principio como movimientos sociales, están aproximando sus funciones a otros grupos, a otros modos de acción colectiva más convencionales. Están llevando acabo un proceso de convencionalización institucional. Por tanto, y para evitar confusiones, conviene separar ambos aspectos. Ver primero el aspecto mas estático, la forma/institución, y describir más tarde la dimensión dinámica, las transformaciones funcionales, el proceso de institucionalización.

\section{LA FORMA/INSTITUCIÓN}

\section{NARRACIONES Y RUTINAS}

Los movimientos sociales son instituciones. Porque son espacios definidos de cómo ver y actuar en el mundo. Un movimiento social es un sistema de relatos, otro sistema más de registros, explicaciones y prescripciones culturales de cómo se expresan determinados conflictos en la sociedad y cómo, a través de qué reglas, ésta debe ser restaurada; cómo el orden, o más exactamente el 
concreto orden, siempre pospuesto y frustrado, de la modernidad debe ser nuevamente reconducido (Thomas et al., 1987). El actor colectivo constituido como movimiento social no actúa en medio de un contexto institucional, diferenciándose nítidamente de ese contexto. Lo que hace un movimiento social, como cualquier otro actor colectivo, es inherente a la definición social de ese mismo actor. Y una definición social de lo que es, debe y puede ser ese actor colectivo es una institución (Thomas et al., 1987: 29-32).

Construir un movimiento social es un extremo acto de libertad colectiva. Pero es un acto que nace y se expande dentro de unos esquemas mentales de conocimiento, valoración y afecto que al preexistir, y percibirse como naturales, inevitablemente estructuran y determinan las opciones y límites de ese nacimiento y posterior desarrollo. No es, pues, una institución desde la perspectiva material, organizativa; pero sí lo es desde el enfoque cultural, esto es, desde un sistema de creencias y códigos que fijan la realidad.

En un movimiento social se pueden discutir y cambiar medios de acción, sistemas de toma de decisiones, incrementar o disminuir las posibilidades de participación de sus miembros, o intensificar en un momento la reivindicación de sus intereses y reforzar en otro sus rasgos identitarios. Sin embargo, todo esto tiene lugar dentro de una cultura establecida, dentro de una institución, pues, como afirma Howarth (1997: 140), una institución es un discurso sedimentado. Ello implica que aquellos que optan por tomar parte en un movimiento social saben de antemano que están eligiendo una determinada formacultura, un estilo - y límites - de juego, asentados a lo largo del proceso de modernización precisamente como una de las formas de canalizar los conflictos provocados por unas específicas exigencias de progreso y justicia ${ }^{3}$.

Desde esta dimensión estática, los movimientos sociales no son tan distintos a otros actores colectivos (partidos y sindicatos) y las mismas instituciones políticas. Son, en todos los casos, instituciones. Esta afirmación es especialmente chirriante si homologamos a los movimientos sociales con las instituciones políticas, dado que precisamente constituye una de las señas de identidad de algunos movimientos sociales su enfrentamiento a las mismas. Pero en la medida que existen distintos planos institucionales, su estrategia de confrontación no desvirtúa la afirmación anterior.

Por un lado, movimientos sociales e instituciones políticas son definiciones estables de la realidad que incluyen espacios y reglas de juego a los que, consciente o inconscientemente, se someten sus participantes. Son, sin duda, espacios y reglas distintos, pero el juego, en última instancia, no es muy distinto. Todo él gira en torno al sueño, o pesadilla, de la modernidad.

Lo que, por otro lado, sí es distinto entre los diversos actores colectivos es la intensidad de la presión y rigidez de los registros y reglas de estas culturas

3 Los movimientos que luchan contra el progreso y la justicia son también movimientos sociales. Estos movimientos, denominados — no siempre de forma correcta—, contra-movimientos, son asimismo un producto, una consecuencia del proyecto de la modernidad. 
convertidas por su estabilidad y horizonte en instituciones. Así, en el caso de los movimientos sociales esta intensidad es menor, lo que permite a los que en ellos participan tener un mayor margen de actuación a la hora de marcar las fronteras; a la hora de distinguirse, o de construirse un mundo en el que se sientan o se vivan como distintos de los demás actores colectivos. Así, convendría en este punto no sobredimensionar los efectos de esta institucionalización. No confundir el que, por un lado, todos los actores colectivos provengan y coincidan tanto en el gran relato y difuso destino de la modernidad y en esa particular forma de ser institución que consiste en describir la realidad y al mismo tiempo crearla con, por otro lado, que todos pretendan hacer siempre lo mismo. Así, partidos, instituciones políticas y movimientos buscan distintos intereses y, por supuesto, los últimos tienen mucho más margen para diseñar y modificar sus tácticas, estrategias y sistemas de comprensión, reconocimiento e identificación colectivos.

Afirmamos anteriormente que los movimientos sociales fueron y son una predeterminada forma de canalizar los conflictos dentro de la modernidad. No obstante, tal afirmación acerca de la modernidad nos permite proponer la idea de la existencia de diferencias entre los movimientos sociales clásicos y los nuevos movimientos por la solidaridad.

Los movimientos sociales son al mismo tiempo forjadores de la modernidad y su propio producto. Expanden la cultura política moderna en cuanto imponen el protagonismo del papel propio, el deseo político — «cívico»- de los ciudadanos de decidir voluntariamente por qué, cómo y cuándo deben organizarse en aras a defender sus intereses colectivos y, eventualmente, transformar la sociedad y el propio poder político.

Y, a su vez, los movimientos sociales son evidentemente moldeados por la modernidad. La dimensión construida libremente, acordada, voluntaria y consciente, característica de una gran parte de las instituciones de la modernidad, conforma asimismo los movimientos. Con la irrupción de la modernidad, los movimientos dejan de ser comunitarios (culturalmente predeterminados por la tradición, espontáneos en la acción, informales, vitalmente radicales con objetivos difusos y defensivos) para convertirse en sociales (más conscientemente construidos, organizados, con intereses definidos que son perseguidos en base a un plan predeterminado) (Tilly, 1978; Della Porta y Diani, 1997: 176). Sin embargo, sostenemos que en todo el proceso de la modernidad el paso de la comunidad a la sociedad nunca fue total; los movimientos sociales de la modernidad mantienen una dimensión comunitaria dentro de su opción social. Es hoy en día, con la postmodernidad, cuando se ha consumado la ruptura (al menos con la dimensión tradicional de la comunidad); cuando la lógica —individual y colectiva - que define y prefigura la acción colectiva de los movimientos sociales es «social», consensuada, en sus términos esenciales ${ }^{4}$.

${ }^{4}$ En realidad, sería más acertado afirmar que lo que ha cambiado es el cómo el individuo se integra en la comunidad (Gurrutxaga, 1996). Durante la premodernidad la persona se adhiere a 


\section{LAS FUNCIONES}

\section{A) SER UNA INSTITUCIÓN PARA SENTIRSE SEGURO}

\section{Certidumbres e incertidumbres en los movimientos por la solidaridad}

Conectando con lo que acabamos de decir, un movimiento social es una institución en cuanto que construye una serie de reglas de comportamiento, o sedimenta un conjunto de rutinas que reducen la incertidumbre en el comportamiento. Como dice Gil Calvo, una institución es un orden natural de comportamiento (Gil Calvo, 1995: 168) o, más exactamente, añadiríamos nosotros, es una percepción colectiva de un orden natural de comportamiento. Las normas de comportamiento se perciben como fijas, como despegadas e invulnerables a las conductas de los que participan en esas instituciones. Por eso las instituciones transmiten esa seguridad; sus miembros se someten a unas rutinas, a unas convenciones preestablecidas y no viven la incertidumbre de tener que decidir o renegociar sistemáticamente las conductas, proyectos o estrategias marcados por la institución.

Y los movimientos sociales participan en cierta medida de esta dimensión institucional. Efectivamente, aun en los momentos constitutivos, en el emocionante estado naciente (Alberoni, 1977) de un movimiento en el que parece que todo se cuestiona y todo se inventa, ese movimiento emergente se conforma a partir de unas tradiciones "movimentistas», a partir de una preexistente cultura/movimiento. Los primeros militantes de un movimiento social, aunque no lo expresen o reconozcan fehacientemente, saben, y eso les otorga seguridad, que están repitiendo reglas, conductas y, en muchos casos, conjuntos de creencias disponibles desde tiempos remotos en la memoria colectiva. Existe un frame/movimiento con el que se alinea el concreto movimiento emergente. Adaptan su grupo a esa forma/movimiento a la que antes hacíamos referencia, a ese movimiento definido estáticamente como institución.

las inamovibles tradiciones de la comunidad (o, más exactamente, es simplemente incluido dentro de ellas). Ya en la modernidad, el individuo decide si quiere formar parte y construir un "Nosotros» comunitario, para lo cual selecciona una serie de rasgos identitarios tradicionales (a este respecto, cabría citar como ejemplo evidente los movimientos nacionalistas). Y, por último, en la era postmoderna se da una persistencia de las demandas de significado, de autoidentificación colectiva, pero que tienden a ser cristalizadas en "comunidades» (si podemos seguir refiriéndonos a ellas como tales) de baja densidad, coyunturales, superpuestas y compartidas con otras identidades colectivas y, en cualquier caso, siempre subordinadas a la centralidad de la esfera privada.

Esta última evolución podría ser parcialmente explicada por la distinción (o, más exactamente, una de las distinciones) existente entre lo público y lo privado. Como afirma Weintraub (1997: 10-16), una de las acepciones de «lo público» alude a las actividades participativas desarrolladas por los ciudadanos (revestidas de virtud cívica) que se sienten miembros de una comunidad, y que actúan siguiendo las orientaciones de esta pertenencia. Por contra, «lo privado» sería la acción del individuo que (uniendo o no sus intereses a los de otros) persigue sus objetivos sin tener como referencia central esa dimensión de la «ciudadanía comunitaria». 
Por otra parte y en etapas sucesivas, con la consolidación del movimiento, la entrada de nuevos miembros supone la aceptación de un conjunto de normas ya establecidas ; de ahí que el acceso al movimiento es un acto que implica menos incertidumbre que el desarrollado por los fundadores del mismo.

Esto dicho, hay que establecer las correspondientes reservas. Porque sólo en cierta medida un movimiento social es un espacio de seguridad. Un movimiento social es también la cristalización colectiva de un conjunto de voluntades decididas a asumir riesgos. Participar en un movimiento social tiene una dimensión creativa difícil de encontrar en otras instituciones más formalizadas. En un movimiento no sólo es posible, sino que constituye un práctica cotidiana, la renegociación colectiva de identidades, intereses y normas de conducta individual y colectiva. Esa flexibilidad, esa apertura, esa neófita sensación de que ahí sí existe la "aventura", la posibilidad de cambiar todo (al margen de que ello realmente sea cierto), es lo que caracteriza a un movimiento social. Un movimiento social surge, pues, en el terreno de la opción por la diferencia y al mismo tiempo constituye una indiscutible terapia a las angustias de la soledad, al pánico de afrontar sin normas previas los retos de la cotidianeidad ${ }^{6}$. Un movimiento social trata de equilibrar estas dos tendencias. Y sólo el mantenimiento de ambas permite su continuidad.

No obstante, acercándonos ahora a los movimientos por la solidaridad, parecería que en éstos el equilibrio aparece inclinado hacia la dimensión de la seguridad. Por el momento, nos conformamos con subrayar el hecho de que tales movimientos surgen, ya desde el comienzo, con un alto nivel de predeterminación en las rutinas colectivas, dejando un escaso margen de riesgo en lo que atañe al diseño identitario o estratégico.

\section{B) SER UNA INSTITUCIÓN PARA CAMBIAR EL MUNDO}

\section{Progresismo y conservadurismo en los movimientos por la solidaridad}

Ahora hemos de considerar si los movimientos sociales juegan el papel, otorgado a las instituciones, de equilibrar la incertidumbre y caos potencial generado por los procesos de elección racional. El neoinstitucionalismo (March y Olsen, 1989; Powell y Dimaggio, 1991; Colomer, 1990) constata, y propugna al mismo tiempo, el liderazgo de las instituciones en ordenar, o al

5 Uno de los rasgos distintivos de los movimientos sociales, su carácter informal, implica normas diferentes de las que caracterizan a otros actores colectivos e institucionales, pero en ningún caso significa una ausencia de normas.

${ }^{6}$ Es este carácter mixto de los movimientos sociales una de las características que les diferencian de otros actores colectivos. Esto es especialmente evidente con respecto a instituciones espontáneas (pongamos por caso la familia) donde la variable de la seguridad es omnipresente. Un movimiento social forma parte de un orden más marcadamente estratégico, donde es posible una mayor creatividad. 
menos reducir significativamente, el desorden provocado por la incoherencia sistemática, por la imposibilidad de lograr una racionalidad colectiva a partir de la simple expresión de múltiples actos de elección racional. No se trata desde este enfoque normativo de proponer un orden social en el que se elimine la libertad del mercado como espacio que, a través de las decisiones de sus concurrentes, concede satisfacción a los diferentes intereses en juego. De lo que se trata es de reforzar el papel que ostentan las instituciones como encauzadoras (no siempre a través de procesos expresamente planificados) de esa tendencia a la dispersión, de esa vulnerabilidad del orden social derivado de la imposibilidad de que decisiones fundamentadas en la elección racional puedan autorregularse hacia una composición más o menos estable del interés general. Es la estructura institucional (Giddens, 1984) la que, a través de un proceso de sedimentación y constitución como memoria colectiva de la repetición de roles individuales, establece un conjunto de normas en un delimitado campo de juego en el que el individuo puede componer racionalmente sus intereses y aun sus identidades, sin que ello destruya ese orden. Todo lo contrario. El mantenimiento del orden social, más exactamente de la continuidad social, sólo es posible a través de la continuidad institucional.

Más todavía, en la medida que las instituciones expresan —a través de esa normativización de la memoria colectiva- el interés general, sólo su continuidad permite, si no garantizar, sí impulsar una continuidad social dirigida hacia el progreso. Parece, pues, que desde este enfoque el incierto futuro del Progreso depende de las instituciones.

Este punto de vista funcional nos permite presentar un par de breves reflexiones sobre los movimientos sociales.

En primer lugar, parece confirmarse esa orientación progresista del neoinstitucionalismo en el caso de los movimientos sociales. Ciertamente, si ha existido en la historia una institución depositaria y reproductora de una memoria colectiva impulsora del progreso, han sido los movimientos sociales. Es en este tipo de institución donde resulta más evidente que la continuidad social propuesta no es simple reproducción del orden establecido, sino impulso y práctica de aquellos valores que, al menos históricamente, ha definido la ideología del progreso; igualdad y libertad y, más recientemente, la defensa de lebenswelt, del mundo-de-vida (Habermas, 1987), como otra forma de luchar por la libertad.

Sin embargo, este liderazgo histórico de los movimientos sociales en impulsar la continuidad progresista de la sociedad ha de ser matizado por lo que respecta a la actualidad. Y otra vez nos referimos a esos recientes movimientos a los que hicimos mención en el apartado anterior.

Nos preguntamos si, por el contrario, aunque sea de forma inconsciente, nuestros movimientos sociales por la solidaridad no están —en la práctica- realizando una función de equilibrio social, de regulación del mercado (en este caso del mercado de sentidos), no tanto dirigida a la continuidad progresista de la sociedad sino a la estricta conservación del orden social establecido. Asimismo, podemos aventurar que algunas instituciones (especialmente políticas y comuni- 
cativas) están promoviendo el liderazgo de estos originales movimientos sociales en la medida que los mismos promueven unos valores y exigen unos cambios en un terreno de juego que en principio no parece cuestionar el orden - los valores dominantes y el sistema de asignación y distribución de recursos- establecido en el territorio controlado por esas mismas instituciones. Así, ese desasosiego social generado por la imposibilidad de armonizar los distintos actos electivos (de valores, sentido o cosmovisiones en este caso) se encauzaría, se desviaría hacia espacios menos peligrosos para la controlada reproducción del sistema.

En este punto, hay que recordar que el conjunto de los movimientos sociales juega en la práctica, al margen de lo que sus objetivos expresos puedan ser, una función sistémica. Pueden, en efecto, hacer posible la mediación entre instituciones políticas y una población fragmentada, impredecible y potencialmente hostil. Como destacan Neidhart y Rucht, el surgimiento de los movimientos sociales «está relacionado con las disfunciones y déficits de la representación de intereses a través de los partidos y grupos de presión» (1991: 448-449).

Si esta aserción es cierta, mucho más lo es la afirmación de que los movimientos por la solidaridad cumplen una función integradora, en cuanto canalizan ciertos problemas sociales hacia un conjunto de demandas que sólo de manera muy indirecta cuestiona los referentes centrales del sistema. Así, si la mediación de los movimientos sociales en general puede ser funcional para el sistema, en el caso de los movimientos por la solidaridad el contenido mediador tiene un potencial integrador y también, en nuestra opinión, ciertas consecuencias concretas y medibles.

\section{C) SER UNA INSTITUCIÓN PARA SER DIFERENTE}

\section{Lo alternativo y lo convencional en los movimientos por la solidaridad}

En este punto de nuestra argumentación debemos ofrecer una más detallada descripción de los movimientos por la solidaridad. Si hasta el momento hemos visto que sus funciones significan un reajuste del habitual papel desarrollado por el conjunto de los movimientos sociales, observaremos ahora que en sus labores más pragmáticas, en su identidad y movilización cotidianas, van más allá de este simple reajuste de las tendencias de los demás movimientos. En cierta forma son funcionalmente diferentes de éstos. No son instituciones alternativas ${ }^{7}$, sino que más bien asumen -aunque no de idéntica forma- cier-

El estudio de los movimientos sociales a través del enfoque de los nuevos movimientos ha destacado cómo éstos construyen densas identidades alternativas.

Los movimientos construyen, enmarcan y definen - la «norma emergente» (Turner y Killian, 1987) — su mundo y el mundo de los demás (Stzompka, 1995: 318). Establecen otras fuentes de información e identificación y definen su papel así como sus guías para una intervención visible; ello constituye una nueva forma tanto de institucionalizar intereses como de introducirlos en el espacio de confrontación público (Maheu, 1995: 13; Eder, 1998). 
tas características y dinámicas típicas de otras instituciones más convencionales que comúnmente denominamos grupos de interés.

Insistiremos en que este parecido con los grupos de interés tan sólo se da en una cierta medida. En sentido estricto, y en oposición a los movimientos sociales, los grupos de interés muestran, entre otras, las siguientes características: una identidad colectiva inexistente o muy débil, una estrategia de cooperación, empleo de medios de acción convencionales, organización formalizada, representación de intereses colectivos definidos y delimitados, y aceptación del sistema en sus normas, funciones y límites ${ }^{8}$. Como veremos más abajo en la tabla comparativa, estas diferencias no son siempre evidentes.

Para continuar, estimamos que merece la pena presentar un cuadro comparativo que sintetiza los dos tipos de movimiento. En otros términos, se desarrolla en él una comparación entre los rasgos característicos de los movimientos sociales globalmente considerados y aquellas especificidades de los movimientos por la solidaridad.

\begin{tabular}{|c|c|c|}
\hline & Conjunto movimientos sociales & Movimientos por la solidaridad \\
\hline $\begin{array}{l}\text { CARÁCTER DEL } \\
\text { BIEN CONSTRUIDO }\end{array}$ & $\begin{array}{l}\text { El bien construido es común/ } \\
\text { solidario por como se exige y por } \\
\text { como se propone su disfrute }\end{array}$ & $\begin{array}{l}\text { El bien construido es común/solida- } \\
\text { rio por como se exige y por como se } \\
\text { propone su disfrute }\end{array}$ \\
\hline INTERESES & Indeterminados & Indeterminados \\
\hline REPRESENTADOS & $\begin{array}{l}\text { Miembros del grupo y otros. } \\
\text { Tendencia hacia una mayor inde- } \\
\text { terminación y globalidad }\end{array}$ & $\begin{array}{l}\text { Otros. } \\
\text { Tendencia hacia una mayor } \\
\text { determinación y sectorialización }\end{array}$ \\
\hline $\begin{array}{l}\text { IDENTIDAD } \\
\text { COLECTIVA }\end{array}$ & $\begin{array}{l}\text { Fuerte; con tendencias exclusivas } \\
\text { y totalizadoras; más expresiva }\end{array}$ & $\begin{array}{l}\text { Menos densa, con tendencia a ser } \\
\text { compartida con otras identi- } \\
\text { dades; menos expresiva }\end{array}$ \\
\hline RIESGO & $\begin{array}{l}\text { Riesgo asumido. Proceso perma- } \\
\text { nente de transformación identita- } \\
\text { ria }\end{array}$ & $\begin{array}{l}\text { Identidad definida desde el } \\
\text { comienzo; escaso riesgo de trans- } \\
\text { formación }\end{array}$ \\
\hline FRAME CULTURAL & $\begin{array}{l}\text { Ideologías/discursos alternativos } \\
\text { (o sedimentos ideológicos...) }\end{array}$ & $\begin{array}{l}\text { Ideologías/discursos inexistentes, } \\
\text { pero conjunto de creencias críticas } \\
\text { acerca de la sociedad }\end{array}$ \\
\hline ENEMIGO & Definido y visible & Difuso \\
\hline ORGANIZACIÓN & Informal & Formal \\
\hline
\end{tabular}

${ }^{8}$ Para un análisis de las diferencias entre movimientos sociales y grupos de interés, consúltense Wilson (1998) e Ibarra y Letamendía (1998). 


\begin{tabular}{lll}
\hline MEDIOS & $\begin{array}{l}\text { Redes/familia } \\
\text { Horizontales } \\
\text { Participativos } \\
\text { No-convencionales }\end{array}$ & $\begin{array}{l}\text { Horizontales } \\
\text { Participativos } \\
\text { Convencionales }\end{array}$ \\
\hline ESTRATEGIA & Conflicto & $\begin{array}{l}\text { Cooperación (conflicto no exclui- } \\
\text { do en principio) }\end{array}$ \\
\hline $\begin{array}{l}\text { POSICIONAMIENTO } \\
\text { ANTE EL SISTEMA }\end{array}$ & $\begin{array}{l}\text { Cambiar las estructuras que gene- } \\
\text { ran la injusticia }\end{array}$ & $\begin{array}{l}\text { Cambiar la coyuntura; paliar los } \\
\text { efectos producidos por la injusticia }\end{array}$ \\
\hline
\end{tabular}

Antes de seguir adelante, una precisión resulta obligada. La expresión "conjunto de movimientos sociales» exige una explicación. Efectivamente, no todos los movimientos sociales que en principio se incluyen en tan genérica descripción tienen en todas sus expresiones y en todos sus momentos las características descritas. Hay coyunturas en las que el conjunto de todo un movimiento tiende a acercarse a los rasgos de nuestros movimientos por la solidaridad (lo que en cualquier caso, como veremos, es distinto a elegir, desde el origen, tales rasgos). Y, asimismo, hay concretas organizaciones de un movimiento social, entendido éste como red, como familia (Della Porta y Rucht, 1995), como en la práctica son los movimientos sociales, que adoptan los rasgos de los movimientos por la solidaridad. Por tanto, nuestra expresión implica un acercamiento, una definición estática de los movimientos sociales, en los que en cierto modo se fijan, en su proceso evolutivo, tendencias originales y asimismo se priorizan las posiciones de los grupos más informales de la red'.

Así, las diferencias que vamos a resaltar a continuación deben ser vistas sólo bajo este prisma general. Si no se hace así, si lo que se tiene en mente a la hora de visualizar las diferencias es un concreto momento de un concreto movimiento, o una específica organización de un movimiento, puede ocurrir que no se detecte ninguna divergencia.

En todo caso, no es nuestra intención describir en profundidad todas estas

9 Adentrándonos en este arriesgado terreno de las definiciones, especialmente en un fenómeno social que, por esencia, está en continuo movimiento, en permanente cambio, apunto una definición, basada en otra que hice en otro lugar (Ibarra y Letamendía, 1998) y en la que trato de incorporar una visión más dinámica. Así, un movimiento social sería: Red de interacciones informales entre individuos, grupos, y/u organizaciones que, en sostenida y habitualmente conflictiva interacción con autoridades políticas, élites y oponentes, y compartiendo una identidad colectiva en origen diferenciada — pero en su proceso evolutivo pueden confundirse con identidades convencionales del "mundo exterior»-, demandan públicamente cambios (tendencialmente antisistémicos) en el ejercicio o redistribución del poder en favor de intereses cuyos titulares son indeterminados e indeterminables colectivos o categorías sociales. La definición es una mezcla — no demasiado ortodoxade los conceptos de Tilly (1993), Diani (1992) y Tarrow (1997) a la que he añadido la dimensión evolutiva. 
diferencias. Algunas de ellas han sido explicadas en detalle y otras caben ser deducidas directamente del cuadro. Sin embargo, sería útil hacer alguna precisión especialmente para reafirmar lo que ya hemos expresado. Queremos insistir en que los movimientos por la solidaridad muestran ciertos rasgos similares a los de los grupos de interés, pero también quisiéramos destacar que comparten ciertas características con lo que hemos denominado el conjunto de los movimientos sociales (las características que aparecen en cursiva) y, al mismo tiempo, en algunas de las categorías las diferencias no son taxativas, sino sólo de intensidad. Así, examinando las variables, subrayaríamos las siguientes:

- Respecto al concepto de bien que es construido y a los intereses representados, prevalecen las similitudes sobre las diferencias. Los movimientos por la solidaridad también defienden que solamente desde la práctica de la solidaridad es posible demandar el bien, y extienden esta definición de la solidaridad a la forma en que el bien buscado debe ser disfrutado; el desarrollo económico de los desfavorecidos, la paz, etc., son bienes para la comunidad, que no pueden ser divididos ni distribuidos.

Lo mismo vale para los intereses representados; independientemente de si el proceso de evolución tiende hacia una mayor determinación y sectorialización o no, es evidente que no constituyen la agregación de concretos y delimitados intereses de los miembros del movimiento, a la manera en que aparecen los grupos de interés.

- La distancia se incrementa en las dos categorías siguientes. Como hemos afirmado, estos movimientos son tan sólo «formalmente» comunitarios, siendo su identidad débil y compartida con otras identidades colectivas o individuales. Como resultado de ello, no son movimientos con una excesiva vocación comunitaria; aceptan como algo natural la diversificación y atomización de la sociedad actual y no persiguen el recrear el mundo a imagen y semejanza de su identidad colectiva y su comunidad.

- En las cuatro categorías siguientes, las similitudes y diferencias están mezcladas. Los movimientos por la solidaridad mantienen algunas — aunque muy genéricas - convicciones críticas; no se organizan en forma jerárquica (para ellos la participación es una opción racional), pero tienden a elegir medios convencionales y su estrategia básica es la de cooperación.

- Finalmente, quisiéramos recordar algo que ha sido ya referido en el lugar adecuado: estos movimientos no son antisistema. No sería adecuado afirmar que son conservadores, pero sus reivindicaciones y sus prácticas no cuestionan las estructuras básicas del sistema. En resumidas cuentas, esto explica por qué no se sienten especialmente incómodos o frustrados por la absorción de sus demandas por instituciones y partidos; consideran esto como un factor positivo para la defensa de sus intereses. 


\section{La evolución del contexto}

Ahora debemos considerar qué transformaciones han tenido lugar en el contexto cultural y político que han hecho posible y han promovido la aparición y expansión de estos movimientos.

Tales transformaciones han afectado, en última instancia, a las dos grandes dimensiones o variables de los movimientos (y, a su vez, las dos más tradicionales formas de aproximación analítica): la variable constructivista y la contextualista $^{10}$. Sin duda, muchas de las consideraciones teóricas que deduciremos de estas variables son bien conocidas, pero estimamos que una síntesis ordenada, y ocasionalmente una profundización de la misma, nos puede ser útil para enfocar mejor el problema.

\section{- Variables constructivistas.}

Como es bien conocido, éstas aluden a la identidad colectiva del movimiento y su potencial movilizador (McAdam, 1988; Gamson, 1992; Maiz, 1995). Explican las posibilidades de éxito del movimiento en su tarea identitaria dependiendo de hasta qué punto su discurso formador de la identidad coincide con el frame cultural dominante en la sociedad. Dicho de otra forma, dependiendo de en qué medida los movimientos pueden utilizar este frame a su favor.

Operar con el enfoque de los frames, y en este caso con el del master frame (Snow y Benford, 1992; Gerhards, 1995), nos permite, desde la flexibilidad de este concepto, distinguir diferentes, aunque interconectados, master frames. En otros términos, aquellos esquemas mentales preexistentes a través de los cuales los sucesos y mensajes recibidos son comprendidos, clasificados, evaluados y sentidos y, como consecuencia de lo cual, la acción es realizada; tales esquemas son dominantes en cuanto que se encuentran extendidos y arraigados en el conjunto de la sociedad. Distinguiremos tres de ellos que, a su vez, han evolucionado de la siguiente forma:

- Por un lado está aquel que podríamos considerar más cercano a la cultura política. En este caso podríamos distinguir un frame de protesta (McAdam, 1994) y un frame de diálogo. En el primero, la sociedad interpreta como natural la respuesta de la protesta abierta, enfocada hacia la suma cero de intereses en conflicto, y por lo tanto comparte con el movimiento sus prácticas

${ }^{10}$ La conexión existente entre la dimensión constructivista y el contexto político es evidente. Un frame es, entre otras cosas, una forma de percibir el contexto político y éste, a su vez, se reorienta y reubica sobre la base de los frames disponibles (en este sentido, ver Diani, 1996: 1056; Gamson y Meyer, 1996). Es asimismo evidente que la acción de un movimiento crea capital social, extiende relaciones, solidaridades, confianza y, a su vez, este incremento de capital social aumenta simultáneamente los cambios en el contexto cultural y político (Diani, 1997: 1439). 
y horizontes radicales. En el segundo, el frame de protesta es reemplazado por una predisposición hacia el diálogo, la búsqueda no dramática del reajuste pacífico de un nuevo equilibrio de intereses.

- Podríamos relacionar otro frame con la existencia de algunos cleavages fundamentales. Así, es posible detectar la existencia de un master frame radical y otro más difuso. El primero de ellos opera en el nivel social y en el interior de la comunidad nacional/política, cristalizándose la línea divisoria en torno a la injusticia de la desigualdad estructural; la desigualdad entre ricos y pobres, entre poderosos y débiles. El segundo establece unos culpables más distantes y la línea divisoria opera con conceptos morales — «buena y mala gente»—, presentándose así como un frame relacionado con la caridad.

- Un último frame se relaciona con la cuestión bien conocida de los valores materiales y postmateriales (Inglehart, 1991). Sin embargo, en nuestro caso, los frames en competición describen valores postmaterialistas frente a valores post-postmaterialistas (podríamos denominarlos también «neomateriales» o "neoprivados»). Si nos fijamos en las actitudes, en cómo los valores son propuestos, podemos esbozar una cultura postmaterialista caracterizada fundamentalmente por la defensa de la autonomía, el rechazo de la distinción entre esferas pública y privada y una búsqueda del comienzo de una nueva utopía a partir de la experiencia cotidiana de una específica comunidad. Por el contrario, el frame «neomaterial» acepta la división público/privado y renuncia al horizonte utópico, así como a la búsqueda de una comunidad exclusiva. Se da un cierto proceso de privatización y, de alguna forma, de banalización de los valores.

Estimamos que en el caso español los master frames han evolucionado, en los tres casos, hacia el diálogo, la culpabilidad difusa y los valores «neoprivados» ${ }^{11}$, todo lo cual ha favorecido, como veremos, a los movimientos por la solidaridad.

\section{- Variables contextualistas.}

Ahora debemos hacer una breve referencia a la evolución de la estructura de oportunidad política (Kriesi, 1995; Della Porta y Rucht, 1995; McAdam, 1996), utilizando solamente dos de sus variables más operativas. El factor institucional y el sistema de alianzas.

${ }^{11}$ La expresión de estos nuevos frames en el campo de la cultura política, entendida ésta de modo amplio y flexible (Llera, 1997), nos lleva a comprobar, por ejemplo, cómo los ciudadanos asumen cada vez más como comportamiento «natural» la cooperación frente al conflicto y la reforma frente a las transformaciones radicales (así, Moran y Benedicto, 1995; Halman y De Moor, 1994: 45-49; Montero y Torcal, 1990, 1994; para el País Vasco, Elzo, 1992: 57-63, 1996) o simplemente cómo progresivamente se desplazan hacia la derecha en su autoubicación (Tezanos, 1997: 497). 
- En el primero de ellos se ha hecho evidente una mayor apertura. De una forma espectacular en la configuración de la agenda y más moderadamente en las fases subsiguientes del habitual proceso de formación de políticas públicas. El medio ambiente, los derechos de la mujer, la cooperación con el Tercer Mundo, etc., son materias normalmente incluidas en las agendas institucionales $\mathrm{y}$, frecuentemente, desde las instituciones políticas son formuladas peticiones para que los movimientos participen en las fases de identificación de problemas y debate de propuestas. Con todo y con ello, es extraño que los movimientos entren en la más estricta fase de toma de decisiones y, por supuesto, su presencia es prácticamente nula en los momentos de la puesta en práctica y la evaluación de las políticas públicas.

- Respecto a las alianzas, desde una fase de desconfianza mutua e incluso hostilidad entre aliados potenciales (partidos de izquierda y movimientos), se ha pasado a una fase marcada por ciertas relaciones que, si bien se desarrollan en la informalidad, expresan mayor fluidez y comprensión. La consecuencia de este cambio ha sido que los partidos han absorbido en mayor o menor medida las demandas de los movimientos, sin que esto, en contrapartida, haya significado que se les haya otorgado a éstos un estatus de interlocutor o mediador al mismo nivel que los propios partidos.

\section{- Efectos de las variables en los movimientos.}

a) En primer lugar, consideraremos las consecuencias de estas transformaciones de orden cultural y político en el conjunto de los movimientos sociales. Parece claro que la relación más reciente entre las identidades originales y los frames dominantes viene marcada por el conflicto. Se da una imposibilidad o una dificultad seria para que éstos puedan ser utilizadas por aquéllas. La cultura de la protesta y la del cleavage de la desigualdad desaparecen o disminuyen con respecto a los «enemigos» cercanos o identificables. Las propuestas de agitación y movilización efectuadas por los movimientos aparecen en cierta forma como inoportunas. Finalmente, el frame «neomaterial» dominante deja escaso margen para propuestas de carácter globalizador.

Los efectos de la estructura de oportunidad política sobre estos movimientos son los siguientes:

La inclusión de las demandas de éstos tanto en la agenda institucional como en los programas de los partidos hace perder a los movimientos el monopolio de tales reivindicaciones, al competir con recursos más exiguos que otros actores colectivos. De igual forma y como veremos más adelante, sus relaciones con las instituciones debilitan a largo plazo su capacidad de movilización.

En resumen, podemos concluir que tanto las variables constructivistas como las contextualistas han llevado a los movimientos sociales a cierto declive en su proceso cíclico. Ello, entre otros factores, ha provocado un interesante 
fenómeno de movilidad social (el cual tan sólo mencionaremos por razón de espacio): el traslado de militantes de aquellos movimientos (feministas, ecologistas) y de otros movimientos más antiguos (grupos radicales del movimiento obrero) hacia otros, los cleavages que generan los movimientos por la solidaridad.

b) $\mathrm{Si}$ observamos los efectos de las variables en los movimientos por la solidaridad, la relación que se establece es mucho más funcional que en el caso previo. El motivo es que sus identidades originales están mucho más adaptadas tanto al sistema de frames dominantes como a la estructura de oportunidad política.

Éstos son movimientos que emergen con una mayor vocación de diálogo y negociación que de protesta. Movimientos que se alinean con cleavages más distantes y con más difusos enemigos, movimientos, sobre todo, que no proponen un cambio sustancial en las relaciones políticas entre la sociedad y el sistema político. Ciertamente, se esfuerzan en obtener de las instituciones políticas decisiones favorables a sus demandas, pero no buscan competir al mismo nivel que los partidos en los procesos de toma de decisiones; y en mucha menor medida tratan de moldear el sistema de toma de decisiones de acuerdo a una forma alternativa de concebir la sociedad — autenticidad, horizontalidad, globalidad, comunidad, etc.

c) Una buena forma de calibrar las diferencias es la de analizar las diferentes actitudes - y las diferentes consecuencias - a la hora de afrontar los procesos de negociación con las instituciones políticas.

Como ya hemos dicho, en años recientes se ha dado cierta apertura del ámbito institucional a las demandas de los movimientos. Las instituciones políticas buscan la implicación por parte de los movimientos en los procesos de toma de decisiones. Esta demanda de las instituciones recibe, sin embargo, una incierta respuesta por parte de los movimientos sociales "tradicionales». Parecería que la apertura institucional a las demandas de éstos no se corresponde con una mayor utilización por su parte de los diferentes canales de acceso a las instituciones. Esto se debe a lo siguiente.

Como resultado de la reducción del potencial movilizador tanto por la crisis de los frames disponibles como por absorción de sus demandas originarias por instituciones y partidos, los movimientos no encuentran suficientes recursos para imponer la presencia de sus reivindicaciones en los estados más avanzados de la formación de políticas públicas. Si bien desearían estar presentes puesto que no abandonan su orientación hacia el poder (Ibarra, 1995), la forma de hacer ésta eficaz implicaría incorporar a esos espacios político-institucionales sus rasgos de identidad y protesta, el discurso en torno a las desigualdades radicales, la dramatización de los acontecimientos, la defensa de la comunidad «fuerte», etc. No obstante, estas características son más retóricas que prácticas, habiendo perdido su capacidad movilizadora. Por ello, los movi- 
mientos se ven dentro del proceso decisorio sin una fuerza movilizadora exterior que posibilite la presión en el proceso de negociación, lo que puede llevarles a la absorción, a ser convertidos en una institución convencional. De esta forma, los movimientos evitan el acceso a la esfera institucional o, cuando acceden a él, se desmarcan enseguida.

Por contra, los movimientos por la solidaridad no muestran una preocupación excesiva por estar presentes en los procesos políticos de toma de decisiones. No están obsesionados ni en cómo adquirir protagonismo ni en cómo competir. Lo importante para ellos radica en que las instituciones asuman sus demandas, independientemente de si ellos toman o no parte en la correspondiente decisión política.

En síntesis, éstos se adaptan mejor que los anteriores al contexto político y así, a largo plazo, sacan un mejor partido de sus demandas y movilizaciones.

\section{UNA BREVE REFLEXIÓN EN TORNO AL PROCESO DE MOTIVACIÓN}

De acuerdo con Klandermans (1997), el individuo puede tomar la decisión de participar en un movimiento sólo en la medida en que se siente inmerso en una serie de frames de acción colectiva (Gamson, 1992); la participación en este sistema de creencias no implica en sí mismo la opción de participar en el movimiento, pero no "estar» dentro de este sistema de creencias hace impensable una decisión dirigida a la acción colectiva.

Se dan tres variables que dan forma a estos frames. El sentimiento de que algo es injusto y de que hay alguien responsable de tal injusticia; el sentimiento de que ésta oprime al colectivo en el que es sentida la solidaridad, de que existe, por tanto, un "nosotros», una realidad y una identidad colectiva violadas por los «otros»; y, finalmente, el sentimiento de que es posible, juntos y unidos, a través de la movilización colectiva, superar la injusticia.

Refiriéndonos a la primera variable, el sentimiento de injusticia con la correspondiente asignación de culpables, surge de tres posibles circunstancias. La experiencia desde el grupo de una desigualdad ilegítima; injusticias cometidas sobre el colectivo; y violación de valores o creencias compartidas. En el caso concreto de los movimientos por la solidaridad, parece que sólo la tercera circunstancia puede ser detectada. En otros términos, tanto en los movimientos de cooperación internacional, en los movimientos antirracistas y en algunos de los pacifistas, las experiencias personales o grupales que reflejan pérdida o daño continuo o imprevisto no son claramente apreciables.

Por otro lado, el descontento, debida y colectivamente enmarcado, es transformado en acción a través de dos tipos de incentivos: colectivos y selectivos.

El primero de ellos convence al individuo de que su participación es nece- 
saria ${ }^{12}$ para obtener los beneficios colectivos que le parecen relevantes y para los cuales lucha el movimiento. Con respecto al segundo, el individuo decide participar porque el movimiento le ofrece, además de la posibilidad de obtener beneficios colectivos, ventajas personales; tanto materiales — como las publicaciones sólo para miembros- como sociales, como por ejemplo el sentirse reconocido por aquellos que ama o admira (o simplemente ser reconocido por alguien).

En mayor o menor medida, ambos tipos de incentivos están siempre presentes. Pero, en relación con la segunda clase de incentivo selectivo -el de tipo social- y refiriéndonos al proceso motivacional de acceso a nuestros movimientos por la solidaridad, nos preguntamos si no hay un claro desequilibrio en favor de los incentivos selectivos en detrimento de los de carácter colectivo. Por una parte, parecería que la necesidad de obtener el interés general no es vivida con la intensidad que se da en los casos en los que el individuo está personalmente afectado por la ausencia (aplazamiento o pérdida) de tal interés, lo cual plantearía que los incentivos dominantes son los selectivos en su dimensión social. Sin embargo, no debemos sobrevalorarlos: como vimos, los movimientos por la solidaridad muestran una identidad colectiva no muy densa y generalmente compartida con otras identidades, lo cual implica que estos espacios de reconocimiento mutuo no son ni exclusivos ni absorbentes.

\section{LA SEGUNDA HIPÓTESIS}

\section{UN NUEVO ESCENARIO}

Como destacamos en la introducción, el análisis de los movimientos por la solidaridad da lugar a otra suposición analítica consistente en que estamos siendo testigos de una nueva forma de acción colectiva. Estimamos que tal hipótesis puede ser propuesta en la medida que otros conflictos adoptan la configuración de los movimientos por la solidaridad; es decir, no es éste mera-

${ }_{12}$ Creemos que la decisión de participar basada en intereses colectivos cae fuera del dilema de Olson (1968), basado en el estricto cálculo de costos y beneficios que conduce, según esta teoría, a la posición del free rider. Por el contrario, estimamos que, más allá de la oferta de incentivos selectivos, el individuo se decide a participar aunque crea, desde una perspectiva cuantitativa, que las posibilidades de éxito no se incrementan con su participación individual. Como dice Klandermans, "si estuviésemos de acuerdo con el modelo de Olson, tendríamos que considerar que los individuos toman decisiones de forma aislada, como si no existiesen otras personas con las que consulta, vive y comparte su solidaridad; con personas a las que se siente ligado con promesas, por las cuales se siente presionado; como si no existiesen todas esas dimensiones sociales que llevan a la gente a luchar unida».

Precisamente porque la gente es consciente tanto del dilema de la acción colectiva como del riesgo que implicaría para alcanzar un beneficio colectivo el que muchos optasen por la posición del free rider, eligen participar en un movimiento social y tratan de lograr que los otros hagan lo mismo (ver, en este sentido, Ferree, 1992, y Maxwell y Oliver, 1993). 
mente un caso de movimientos con diferentes objetivos y que adoptan diferentes formas, sino más bien un caso de conflictos que anteriormente adoptaban formas correspondientes al comportamiento común del conjunto de los movimientos sociales y que ahora adoptan las formas características de los movimientos por la solidaridad. Consideraremos este nuevo escenario con algo más detalle, insistiendo, y muy especialmente en esta segunda hipótesis, que las siguientes son exclusivamente suposiciones provisionales.

Parece que ha surgido una ruptura y un proceso de reajuste de los instrumentos colectivos que tratan de resolver diversos conflictos y tensiones.

Por un lado, ciertas injusticias cuyas respuestas antes se expresaron en la forma integral de los movimientos sociales adoptan ahora la forma limitada/ parcial de los grupos de interés.

Por otro lado - y ésta es la cuestión que quisiéramos subrayar-, aparece una nueva forma de respuesta a la cual denominaremos la de los nuevos voluntarios. Nuevos por dos razones.

No es igual que la de los anteriores voluntarios en el sentido de que incluyen algunos rasgos de los movimientos sociales que aquéllos ni tenían ni tienen. Por ejemplo, como vimos, la construcción colectiva de un bien común en contraste con la simple adhesión individual a objetivos predeterminados, característica del viejo voluntariado; aunque, como dijimos, en otros aspectos - como la lógica de cooperación frente a la lógica de conflicto- marcan distancias respecto al conjunto de los movimientos sociales...

$\mathrm{Y}$ nuevos sobre todo porque aparecen en otros campos de conflicto que tradicionalmente fueron menos proclives a la acción colectiva de voluntarios. Aunque, ciertamente, su mayor presencia continúa siendo la solidaridad con los marginados de la tierra, sin embargo, también los conflictos derivados del mundo del trabajo, el medio ambiente, el pacifismo, etc., están adoptando desde que inician su andadura movilizadora esta forma híbrida.

Anteriormente se daban dos tipos de lógica dominante en la acción colectiva (Offe y Wiesenthal, 1985). Una de ellas con pretensiones integradoras, globales, simultáneamente construida en la solidaridad material y cultural, como es el caso de los movimientos sociales. Y la otra definida por la agregación de intereses individuales; altruistas en un caso - los viejos voluntariosy estrictamente material/individual en el otro, como es el caso de los grupos de interés.

Hoy en día aparecen otras dos lógicas dominantes. La de los nuevos voluntarios y la de los grupos de interés. Y lo que ha cambiado hoy son las tendencias de la evolución; es decir, cuando anteriormente ciertos conflictos en ciertas coyunturas, más tarde o más temprano, daban paso a un ciclo de movimientos sociales, ahora conflictos y coyunturas similares evolucionan hacia esas otras formas de acción colectiva.

Dicho de otro modo, lo que emerge es un reajuste de las opciones de la acción colectiva, un desplazamiento del protagonismo entre diferentes formas de acción colectiva. 
Nos hemos referido a cómo la transformación de ciertos contextos culturales y políticos favoreció la extensión de los movimientos por la solidaridad. Sugerimos ahora que un cambio cultural más profundo está probablemente detrás de esas transformaciones, un cambio que promueve el protagonismo de esos nuevos voluntarios y de esas nuevas formas de acción colectiva.

$Y$ estimamos que se da un cambio cultural porque hay una transformación de las rutinas institucionales. No hay ya ningún recuerdo de la tradicional rutina/narrativa de la forma institución/movimiento; y esto es así a causa del proceso de sustitución de la sociedad civil por el mercado, por la hiperfragmentación social y la dificultad en construir identidades extensivas y exclusivas; el contexto tiende hacia identidades pequeñas, superpuestas, intercruzadas, intercambiables y no muy densas. Ésta es la razón por la que no hay memoria previa y el contexto no favorece su recuperación. La tendencia es optar por una forma/institución más adaptada a este nuevo orden.

\section{REFUTANDO OBJECIONES}

Para concluir, responderemos a algunas previsibles objeciones.

- La primera de ellas es que no estamos teniendo en cuenta la dimensión cíclica (Tarrow, 1997); que los movimientos sociales surgen y se extienden con rasgos típicamente «movimentistas» y que, posteriormente, en cuanto que adaptan sus demandas al contexto cultural y político, adquieren las características de los grupos de interés. Esta objeción añade que dicho proceso de evolución tendente a la similitud con los grupos de presión es un denominador común prácticamente en todos los movimientos en su fase de estabilización o declive.

No obstante, estimamos que tal objeción no es del todo consistente. Efectivamente, es característico de estos movimientos que desde su propio surgimiento muestran los rasgos que hemos apuntado. El predominio de la seguridad sobre el riesgo innovador en la construcción de la identidad, la aceptación del sistema para no trastocar los equilibrios ni exceder sus límites, la adaptación sin traumas excesivos al dominante master frame cooperativo, etc., son características que aparecen en la fase de surgimiento.

- En segundo lugar, otra posible objeción es que estamos confundiendo la parte con el todo, que estamos describiendo como un conjunto de movimientos lo que no es sino una parte de los mismos: su organización formal. De acuerdo con este argumento y tal como dijimos en su momento, los movimientos sociales son una red de relaciones entre diferentes personas, grupos, movilizaciones no-convencionales, etc., así como, evidentemente, organizaciones formales. Y estas organizaciones formales de los movimientos sociales, de cualquiera de ellos, tienden — a causa del tipo de actividad que desempeñan- 
a asumir ciertas prácticas y una weltanschauung no demasiado alternativas, bastante convencionales y similares a las típicas de los grupos de interés. Como resultado de todo ello — concluye el argumento-, nuestra descripción no se corresponde con el conjunto de las diferentes familias de los movimientos sociales, sino más bien con sus organizaciones concretas, o algunas de ellas.

Sin embargo, tampoco es demasiado sólida esta objeción ya que, como en el caso anterior, lo que es característico de estas formas de acción colectiva es precisamente el hecho de que se expresan casi exclusivamente a través de medios organizacionales; que — quizás expresado de una forma exagerada - la parte es el todo, que el movimiento no es la red de diferentes, sino más bien de iguales formas de acción colectiva, que son sólo (o casi exclusivamente) organizaciones formales. Tampoco creemos que haya habido una evolución por medio de la cual la parte organizacional del movimiento haya estado absorbiendo la identidad colectiva y las prácticas del conjunto; estimamos que lo que es original es que desde el principio han asumido esa perspectiva más formal, más organizativa.

- La tercera y última objeción afirma que lo que estamos afrontando no es una nueva forma de acción colectiva, sino formas que nos son ya conocidas.

Por un lado, podría ser afirmado que estamos tratando con movimientos sociales caracterizados por la práctica del consenso (Schwartz y Shuva, 1992). Sin embargo, en respuesta a este argumento, deberíamos decir dos cosas.

Cuando el consenso es una dimensión constitutiva y permanente de la acción colectiva, tal acción no es un movimiento social en tanto que lo que define a éste es precisamente la dimensión contraria: la estrategia de conflicto.

Si lo que se afirma es que éstos son movimientos en fase de consenso, debemos admitir que, efectivamente, los movimientos sociales pasan por dicha fase, pero tenemos también que reiterar una vez más que estamos hablando de una tendencia en la que el consenso en la acción colectiva es tanto estable como presente desde el comienzo.

Por otro lado, podría ser apuntado que el modelo descrito es el que corresponde a los grupos de interés público (Jordan, Maloney y Bennie, 1996), donde lo característico, como en nuestro caso, es que el bien perseguido tiene un carácter público y general que no está limitado a los miembros del grupo. Sin duda, nuestro modelo propuesto muestra similitudes con estos grupos de interés, pero hay diferencias también en cuanto tienen ciertas formas de actuar y determinadas pretensiones identitarias que, como vimos, los distancian de los grupos de interés.

\section{CONCLUSIONES}

Para concluir, volveremos a los movimientos por la solidaridad y trataremos de dar un enfoque comparativo en algunos de sus rasgos más característicos. 
Nos queda pendiente por preguntarnos si no estamos asistiendo a un fenómeno de acción colectiva que no se corresponde con los movimientos sociales, sino que se halla más cercano a los grupos de interés sin ser equivalente a ellos.

En algunos aspectos estos movimientos por la solidaridad muestran un mayor parecido a los grupos de interés; por ejemplo, mantienen una posición estricta de presión con respecto al poder político y no se les ocurriría nunca que este último habría de ser desarrollado de manera diferente o ser compartido (creencia presente en ocasiones en los movimientos sociales); en este sentido, estos movimientos no apuntan, ni siquiera potencialmente, a objetivos antisistema (ni en contra del materialismo del sistema ni contra la cultura dominante de este último). Su estrategia movilizadora central está mucho más centrada en la cooperación (grupos de interés) que en el conflicto (movimientos sociales). Su estructura organizacional se halla mucho más cercana a la formalidad que a la informalidad y, finalmente, sus repertorios de acción raramente exceden los convencionalmente establecidos.

Sin embargo, hay una relevante dimensión que los separa de los grupos de interés. Sus miembros no buscan beneficios personales como resultado de su actividad; los intereses generales que desean representar y obtener son los intereses de otros. Este aspecto los distancia radicalmente de los grupos de interés (podríamos decir que reduce al absurdo el discurso olsoniano) y los atrae a la esfera de los movimientos sociales. En todo caso, la común búsqueda de la identidad colectiva tiene diferentes consecuencias en los movimientos por la solidaridad y en el resto de los movimientos sociales. En los primeros ello no conduce a la construcción de frames cognitivos y morales en conflicto con los dominantes, mientras que en los segundos tal conflicto es lo habitual.

\section{BIBLIOGRAFÍA}

Alberoni, F. (1977): Movimento e istituzione, Bologna, Il Mulino.

Bárcena, I.; Ibarra, P., y Zubiaga, M. (1995): Nacionalismo y ecología, Madrid, Libros de la Catarata.

Colomer, J. M. (1990): «Retorno a las instituciones», Revista Claves, núm. 15.

Della Porta, D., y Diani, M. (1997): I movimenti sociali, Roma, La Nuova Italia Scientifica.

Della PorTA, D., y Rucht, D. (1995): «Left-libertarian movements in context. A comparison of Italy and West Germany 1965-1990», en Jenkins y Klandermans (eds.), The Politics of Social Protest: Comparative Perspectives on States and Social Movements, University of Minnesota Press-UCL Minneapolis-London.

Diani, M. (1992): «The Concept of Social Movement», The Sociological Review, núm. 38.

- (1996): «Linking mobilization frames and political opportunities: insights from regional populism in Italy", American Sociological Review, vol. 61.

- (1997): «Social Movements and Social Capital: A network perspective on Movement Outcomes», Mobilization, vol. 2, núm. 2.

EDER, K. (1998): «La institucionalización de la accion colectiva; ¿̧hacia un nuevo modelo teórico en el análisis de los movimientos sociales?», en P. Ibarra y B. Tejerina (eds.), Los movimientos sociales en el cambio de milenio. Nuevas opciones en inciertos contextos, Madrid, Trotta. 
Elzo, J. (coord.), y otros (1992): Euskalerria en la encuesta europea de valores, Bilbao, Universidad de Deusto.

Elzo, J. (dir.), y otros (1996): Los valores en la Comunidad Autónoma del País Vasco y Navarra, Vitoria, Servicio de Publicaciones del Gobierno Vasco.

Eyerman, R. (1992): «Modernity and Social Movements», en H. Haferkamp y N. J. Smelser (eds.), Social Change and Modernity, Berkeley, University of California Press.

FERree, M. M. (1992): "The political context of rationality; rational choice theory and resource mobilization», en A. Morris y C. MacCluerg Mueller, Frontiers in Social Movements Theory, N. Haven, Yale University Press.

Gamson, W. A. (1992): Talking politics, Cambridge (Mass.), Cambridge University Press.

GAmson, W. A., y Meyer, D. S. (1996): «Framing political opportunity», en D. McAdam, J. D. McCarthy y M. Zald (eds.), Opportunities, mobilizing Structures and Framing, Cambridge, Cambridge University Press.

Giddens, A. (1984): The constitution of society, Cambridge, Polity.

Gil Calvo, E. (1995): El destino; progreso, albur y albedrio, Barcelona, Paidós.

GERHARDS, J. (1995): «Framing dimensions and framing strategies: contrasting ideal —and real type- frames», Social Science Information, núm. 34 (2).

GurRUTXAGA, A. (1996): «La representación comunitaria: individualidad colectiva, tradición y socialidad privada», en A. Pérez Agote e I. Sánchez de la Yncera (eds.), Complejidad y teoría social, Madrid, CIS.

Habermas, J. (1987): Teoría de la acción comunicativa, Madrid, Taurus.

Halman, L., y De Moor, R. (1994): «Individualización y cambio de valores en Europa y Norteamérica», en J. Díez Nicolás y R. Inglehart (eds.), Tendencias mundiales de cambio en los valores sociales y politicos, Madrid, Fundesco.

HowarTh, D. (1997): «La teoría de discurso», en D. Marsh y G. Stoker (eds.), Teoría y métodos de la Ciencia Politica, Madrid, Alianza.

IBARRA, P. (1995): «Nuevas formas de comportamiento político; los nuevos movimientos sociales», Inguruak, núm. 13, Bilbao.

Ibarra, P., y Letamendía, F. (1998): «Los movimientos sociales», en M. Caminal (ed.), Manual de Ciencia Política, Madrid, Tecnos.

INGLEHART, R. (1991): El cambio cultural en las sociedades industriales avanzadas, Madrid, CIS.

Jordan, G.; Maloney, W. A., y Bennie, L. G. (1996): "Les groupes d'intéret public», Pouvoirs, núm. 79.

Klandermans, B. (1997): The Social Psychology of Protest, Oxford, Blackwell.

Kriesi, H. P. (1995): "The Political Opportunity Structure of New Social Movements: Its Impact on Their Mobilization», en Jenkins y Klandermans (eds.), The Politics of Social Protest: Comparative Perspectives on States and Social Movements, University of Minnesota PressUCL Minneapolis-London.

LACLAU, E. (1990): New Reflections on the Revolution of our Time, London, Verso.

Llera, F. (1997): «Enfoques en el estudio de la cultura política», en Pilar del Castillo e Ismael Crespo (eds.), Cultura Política, Valencia, Tirant lo Blanc.

MAIZ, R. (1995): «La construcción de las identidades politicas», Inguruak, núm. 13, Bilbao.

MCADAM, D. (1988): «Micromobilization, contexts and recruitment to activism», en B. Klandermans, H. Kriesi y S. Tarrow (eds.), International Social Movement Research, vol. I, Greenwich (Con.), Jai Press.

- (1994): "Cultura y movimientos sociales», en E. Laraña y J. Gusfield (eds.), Los nuevos movimientos sociales: de la ideología a la identidad, Madrid, CIS.

- (1996): "Conceptual origins, current problems, future directions», en D. McAdam, J. D. McCarthy y M. N. Zald (eds.), Comparative perspectives on Social Movements, N. York, Cambridge University Press.

Maheu, L. (ed.) (1995): Social Movements and Social Classes, London, Sage.

Marwel, G., y Oliver, P. (1993): The Critical Mass in Collective Action; a Micro Social Theory, Cambridge (Mass.), Cambridge University Press. 
March, J. G., y Olsen, J. P. (1989): Rediscovering Institutions, New York, Free Press.

MeluCCI, A. (1994): “¿Qué hay de nuevo en los nuevos movimientos sociales?», en E. Laraña y J. Gusfield (eds.), Los nuevos movimientos sociales; de la ideología a la identidad, Madrid, CIS.

Montero, J. R., y TorCal, M. (1990): «La cultura política de los españoles: pautas de continuidad y cambio», Sistema, núm. 99.

- (1994): "Cambio cultural, reemplazo generacional y política en España», en J. Díez Nicolás y R. Inglehart (eds.), Tendencias mundiales de cambio en los valores sociales y politicos, Madrid, Fundesco.

MorÁn, M. L., y Benedicto, J. (1995): La cultura política de los españoles, Madrid, CIS.

Neidhart, F., y Rucht, D. (1991): "The state of the art and some perspectives on further research", en D. Rucht (ed.), Research on Social Movement: The State of the Art in Western Europe and the USA, Boulder (Co.), Westview Press.

Offe, C., y Wiesenthal, H. (1985): «Two logics of collective action», en J. Keane (ed.), Disorganized Capitalism, Cambridge (Mass.), MIT Press.

Olson, M. (1968): «The Logic of the Collective Action», Cambridge (Mass.), Harvard University Press.

Powell, W., y Dimaggio, P. (comps.) (1991): The New Institutionalism in Organizational Analysis, Chicago, University of Chicago.

SNOw, D. A., y BENFORD, R. D. (1992): «Master frames and cycles of protest», en A. D. Morris y C. M. Mueller (eds.), Frontiers in Social Movements Theory, New Haven, Yale University Press.

SChWARTZ, M., y Shuva, P. (1992): «Resource Mobilization versus the Mobilization of People: Why Consensus Movements cannot be Instruments of Social Change», en A. D. Morris y C. M. Mueller (eds.), Frontiers in Social Movements Theory, New Haven, Yale University Press.

SwIndler, A. (1986): "Culture in Action: Symbols and Strategies», American Sociological Review, vol. 51.

SzTOMPKA, P. (1995): Sociología del cambio cultural, Madrid, Alianza.

TARrow, S. (1997): El poder en movimiento, Madrid, Alianza.

Tezanos, J. F. (1997): «La evolución de los espacios político-ideológicos en España», en TeZAnOS, Montero y Díaz, Tendencias de futuro en la sociedad española, Madrid, Sistema.

Thomas, G. M.; Meyer, J. W.; Ramírez, F. O., y Boli, J. (1987): Institutional structure. Constituting State, Society and the Individual, Beverly Hills, Sage.

Tilly, Ch. (1978): From mobilization to revolution, Reading (MA), Addison-Wesley.

- (1993): «Social Movements as Historically Specific Clusters of Political Performances», Berkley Journal of Sociology, núm. 38.

Turner, R., y Killian, L. (1987): Collective Behaviour, Englewood Cliffs (NJ), Prentice-Hall.

Weintraub, J. (1997): "The Theory and Politics of the Public/Private Distinction", en J. Weintraub y K. Kumar (eds.), Public and Private in Thought and Practice, Chicago, The University of Chicago Press.

Wilson, F. L. (1990): «Neo-corporatism and the Rise of New Social Movements», en R. J. DALTON y M. Keuchler, Challenging the Political Order: New Social and Political Movements in Western Democracies, Cambridge, Polity Press. 


\begin{abstract}
This paper contends that the social movements which strive towards solidarity — cooperation with the Third World, anti-racism, etc. - are characterised by certain behavioural patterns as opposed to the patterns that characterise social movements as a whole. Likewise, the author formulates the hypothesis that these different forms of action also prevail in other social spheres (work, ecology, etc.) and that therefore the subject under study constitutes a specific social movement, but may also correspond to a new and generic form of collective action.
\end{abstract}




\section{TEXTO CLÁSICO}

